\section{International Scientific Journal Theoretical \& Applied Science}

\author{
p-ISSN: 2308-4944 (print) e-ISSN: 2409-0085 (online) \\ Year: $2016 \quad$ Issue: $11 \quad$ Volume: 43 \\ Published: 02.11.2016 http://T-Science.org
}

\section{Denis Chemezov}

Master of Engineering and Technology, Corresponding Member of International Academy of Theoretical and Applied Sciences, Lecturer of Vladimir Industrial College, Russian Federation chemezov-da@yandex.ru

SECTION 7. Mechanics and machine construction.

\title{
A CHOICE OF THE GEOMETRY OF THE CYLINDRICAL BROACH'S CUTTING PART DURING A PROCESSING OF STEELS AND CAST IRONS
}

Abstract: The article is presented the calculation of parameters of the geometry of a cylindrical broach's working part, depending on the processed material (grey cast iron, carbon and alloy steels), on the modes and on the cutting forces.

Key words: cylindrical broach, tooth, hole, cutting part.

Language: Russian

Citation: Chemezov D (2016) A CHOICE OF THE GEOMETRY OF THE CYLINDRICAL BROACH'S CUTTING PART DURING A PROCESSING OF STEELS AND CAST IRONS. ISJ Theoretical \& Applied Science, 11 (43): 1-4.

Soi: http://s-o-i.org/1.1/TAS-11-43-1 Doi: crossef http://dx.doi.org/10.15863/TAS.2016.11.43.1

\section{ВЫБОР ГЕОМЕТРИИ РЕЖУЩЕЙ ЧАСТИ ЦИЛИНДРИЧЕСКОЙ ПРОТЯЖКИ ПРИ ОБРАБОТКЕ СТАЛЕЙ И ЧУГУНОВ}

Аннотация: В статье представлен расчет параметров геометрии рабочей части ичллинрической протяжки в зависимости от обрабатываемого материала (серый чугун, углеродистая и легированная стали), режимов и силь резания.

Ключевые слова: ичилиндрическая протяжка, зуб, отверстие, режущая часть.

\section{Введение}

Протяжка представляет собой режущий инструмент для обработки наружных и внутренних геометрических поверхностей различной формы в условиях массового производства [1]. Инструмент является многолезвийным. Последующий режущий зуб протяжки имеет увеличение по высоте по сравнению с предыдущим, равное толщине срезаемого слоя материала заготовки. Для калибрующих зубьев протяжки этот подъем практически отсутствует. Совокупность всех зубьев на протяжке составляют рабочую часть инструмента [2].

Расчет и последующее проектирование конструктивных элементов и геометрических параметров цилиндрических протяжек зависит от ряда факторов: диаметра и длины отверстия в заготовке, материала заготовки, твердости материала заготовки, модели протяжного станка и др. [3, 4] Проектирование усложняется расчетом всех видов деформаций, возникающих при обработке протяжкой. Необходимо учитывать размеры хвостовика протяжки при обработке отверстий различных диаметров [5].

Расчет геометрии рабочей части протяжки позволит проектировать режущий инструмент для обработки цилиндрических отверстий в заготовках из различных металлических материалов с наибольшей производительностью и соблюдением технических требований на изготовление детали.

\section{Материалы и методы исследования}

Выбор геометрии режущей части протяжки осуществлялся для обработки цилиндрических отверстий в конструкционных металлических материалах - углеродистой стали с НВ 120 и 240 (твердостью по Бриннелю), легированной стали с НВ 120 и 240, сером чугуне с НВ 160 и 260.

Процесс протягивания выполнялся на горизонтально-протяжном станке модели 7552 [6, c. 108]. Данный станок предназначен для обработки внутренних поверхностей различной 


\begin{tabular}{|c|c|c|c|c|c|c|}
\hline Impact Factor: & $\begin{array}{l}\text { ISRA (India) } \\
\text { ISI (Dubai, UAF } \\
\text { GIF (Australia) } \\
\text { JIF }\end{array}$ & $\begin{array}{l}=1.344 \\
=0.829 \\
=0.564 \\
=1.500\end{array}$ & $\begin{array}{l}\text { SIS (USA) } \\
\text { PИНЦ (Russia) } \\
\text { ESJI (KZ) } \\
\text { SJIF (Morocco) }\end{array}$ & $\begin{array}{l}=0.912 \\
=0.234 \\
=1.042 \\
=2.031\end{array}$ & $\begin{array}{l}\text { ICV (Poland) } \\
\text { PIF (India) } \\
\text { IBI (India) }\end{array}$ & $\begin{array}{l}=6.630 \\
=1.940 \\
=4.260\end{array}$ \\
\hline
\end{tabular}

формы. Техническими характеристиками станка являются: тяговая сила станка - 100000 кг, наибольший рабочий ход ползуна - 2000 мм, мощность главного электродвигателя - 55 кВт, длина станка - 10315 мм, ширина - 2037 мм, высота - 2415 мм.

Обрабатывались отверстия диаметрами от 25 мм до 100 мм с шагом 5 мм. Длина отверстий в заготовках была одинакова и составляла 200 мм. Размеры обработанных отверстий должны были соответствовать 7-му квалитету точности [7].

Для протягивания на протяжном станке модели 7552 сквозного отверстия Ø35+0.025 мм в заготовке (легированная сталь с НВ 120) общей длиной 100 мм принимается цилиндрическая протяжка с геометрией, представленной на рис. 1.

A
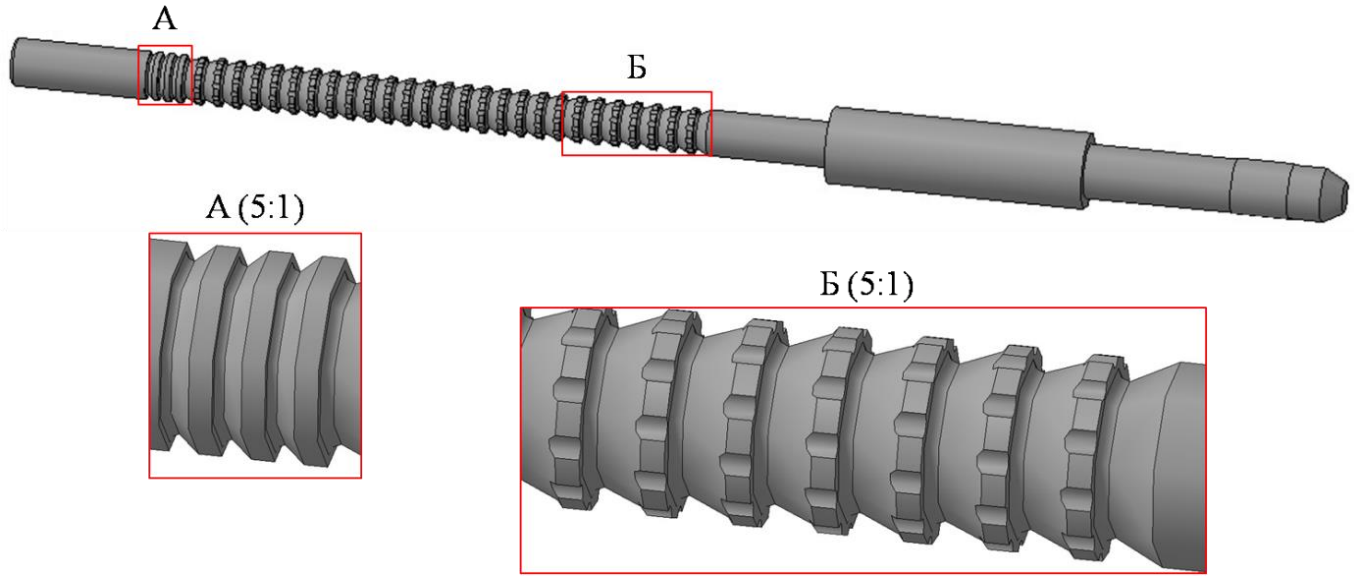

Рисунок 1 - 3D модель цилиндрической протяжки. А и Б - выносные элементы режущих и калибрующих зубьев протяжки соответственно.

Общая длина спроектированной протяжки составила 899 мм (в том числе: длина режущей части - 392 мм, длина калибрующей части - 27 мм). На рабочей части протяжки выполняются 29 режущих зубьев и 3 калибрующих зуба. Глубина канавки между режущими зубьями для размещения стружки принималась величиной 4 мм. Расчетная величина выкружки - 0.15 мм. Хвостовик цилиндрической протяжки для крепления ее в патроне станка принят диаметром 40 мм.

\section{Результаты и их обсуждение}

График зависимостей величины подачи, допустимой по силе резания, от диаметра протягиваемого отверстия в заготовках из серого чугуна, углеродистой и легированной сталей представлен на рис. 2.

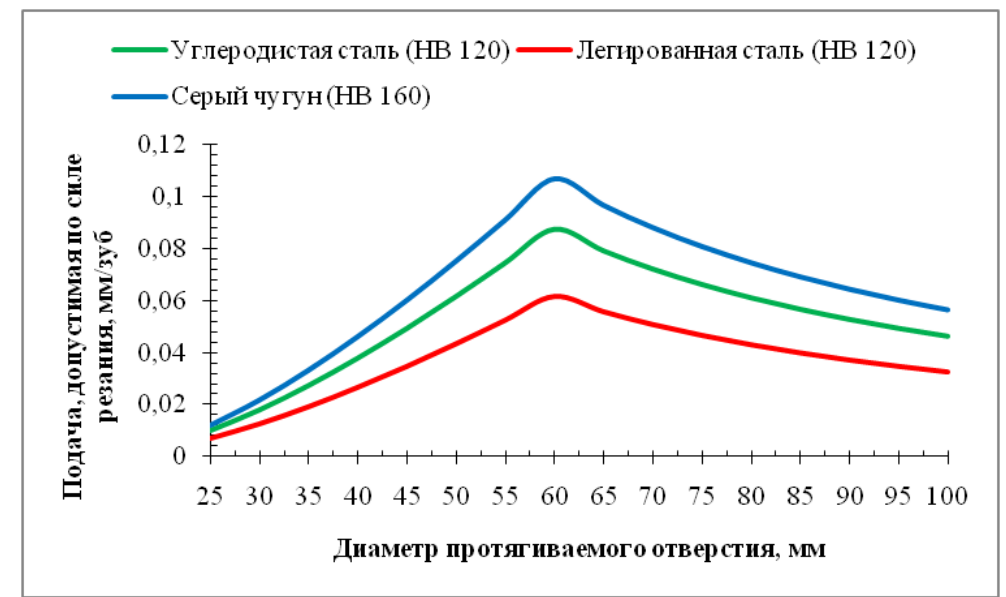

Рисунок 2 - Зависимости величины подачи, допустимой по силе резания от диаметра протягиваемого отверстия.

Подача, допустимая по силе резания больше, чем расчетная подача, допустимая по размещению стружки. В этом случае принимается одинарная схема резания [8].

ISPC Education and Innovation, 
Обработка отверстий диаметром от 25 мм до 60 мм (при глубине стружечной канавки между режущими зубьями протяжки равной 5 мм) характеризуется увеличением подачи, допустимой по силе резания. При обработке отверстий диаметром $60 \ldots 100$ мм предлагается уменьшение подачи на 30 - 40 \%. Наибольшая расчетная подача, допустимая по силе резания (0.107118 мм/зуб) задается при обработке отверстия диаметром 60 мм в чугунной заготовке. Размах изменения расчетной подачи в анализируемом

диапазоне

диаметров протягиваемых отверстий составляет для углеродистой стали - 0.077938 мм/зуб, для легированной стали - 0.054712 мм/зуб и для серого чугуна -0.095139 мм/зуб.

На рис. 3 представлена зависимость величины фактической длины режущей кромки цилиндрической протяжки от наибольшего усилия, допустимого на прочность по 1 зубу в условиях обработки легированной стали с НВ 120.

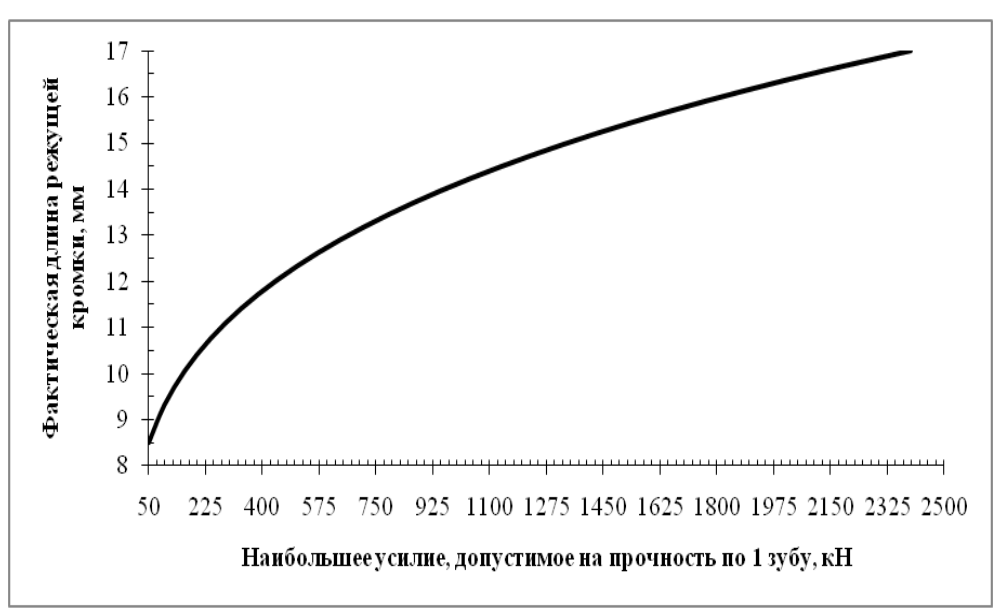

Рисунок 3 - Зависимость величины фактической длины режущей кромки цилиндрической протяжки от наибольшего усилия, допустимого на прочность по 1 зубу.

Величина фактической длины режущей кромки цилиндрической протяжки зависит от диаметра обрабатываемого отверстия заготовки. С увеличением диаметра режущего инструмента возрастает и усилие, допустимое на прочность по 1 режущему зубу протяжки. При увеличении фактической длины режущей кромки протяжки в два раза (с 8.5 мм до 17 мм), усилие, допускаемое на прочность по 1 зубу изменяется в пределах $50 \ldots 2397$ кН.

В табл. 1 приведены результаты расчета параметров рабочей части цилиндрической протяжки при изменении обрабатываемого диаметра отверстия и (твердости) материала заготовки.

Таблица 1

Результаты расчета некоторых параметров рабочей части цилиндрической протяжки.

\begin{tabular}{|c|c|c|c|c|c|c|c|c|c|}
\hline 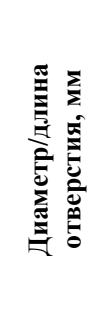 & 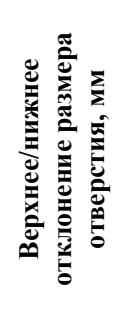 & 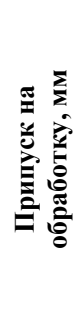 & 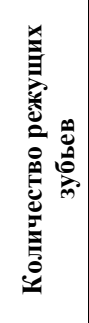 & 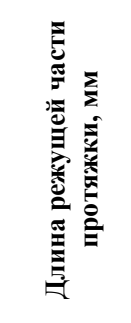 & 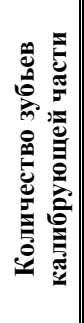 & 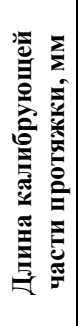 & 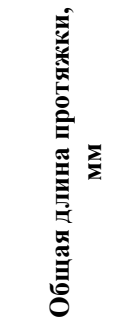 & 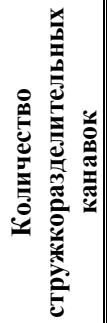 & Вспомогательный угол, град. \\
\hline \multicolumn{10}{|c|}{ Обрабатываемый материал } \\
\hline \multicolumn{10}{|c|}{ "Легированная сталь (углеродистая сталь) НВ 120/240 Серый чугун НВ 160/260 } \\
\hline $30 / 200$ & $+0.021 / 0$ & 2.27 & 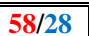 & $1026 / 486$ & 23 & 36 & $1742 / 1202$ & 10 & 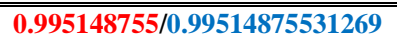 \\
\hline $35 / 200$ & $+0.025 / 0$ & 2.3 & 58/29 & $1026 / 504$ & 3 & 36 & $1742 / 1220$ & 11 & $0.996022897 / 0.994032880807074$ \\
\hline $40 / 200$ & $+0.025 / 0$ & 2.32 & 59/30 & $1044 / 522$ & 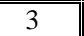 & 36 & $1760 / 1238$ & 12 & $0.996665275 / 0.993327759197324$ \\
\hline$\overline{45 / 200}$ & $+0.025 / 0$ & 2.35 & 60/30 & $\overline{1062 / 522}$ & 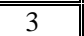 & 36 & $1778 / 1238$ & 12 & $0.997154558 / 0.994307116104869$ \\
\hline$\overline{50 / 200}$ & $+0.025 / 0$ & 2.37 & 60/31 & $1062 / 540$ & 3 & 36 & $1778 / 1256$ & 13 & $\overline{0.99753775 / 0.995074074074074}$ \\
\hline $55 / 200$ & $+0.03 / 0$ & 2.4 & $261 / 31$ & $1080 / 540$ & 3 & 36 & $1796 / 1256$ & 14 & $0.997844618 / 0.995688234499527$ \\
\hline
\end{tabular}

ISPC Education and Innovation, 


\section{Impact Factor:}

\begin{tabular}{lr|lr} 
ISRA $($ India $)$ & $=\mathbf{1 . 3 4 4}$ & SIS $($ USA $)$ & $=\mathbf{0 . 9 1 2}$ \\
ISI $($ Dubai, UAE) $=\mathbf{0 . 8 2 9}$ & РИНЦ $($ Russia $)=\mathbf{0 . 2 3 4}$ \\
GIF $($ Australia) $=\mathbf{0 . 5 6 4}$ & ESJI $($ KZ $)$ & $=\mathbf{1 . 0 4 2}$ \\
JIF & $=\mathbf{1 . 5 0 0}$ & SJIF $($ Morocco $)=\mathbf{2 . 0 3 1}$
\end{tabular}

\begin{tabular}{|c||c||c||c||c||c||c||c||c||c||}
\hline \hline $60 / 200$ & $+0.03 / 0$ & 2.42 & $\mathbf{6 2 / 3 1}$ & $\mathbf{1 0 9 8 / 5 4 0}$ & 3 & 36 & $\mathbf{1 8 1 4 / 1 2 7 6}$ & 14 & $\mathbf{0 . 9 9 8 0 9 4 8 9 7 / 0 . 9 9 6 1 8 9 1 1 1 7 4 7 8 5 1}$ \\
\hline \hline $65 / 200$ & $+0.03 / 0$ & 2.45 & $\mathbf{6 2 / 3 2}$ & $\mathbf{1 0 9 8 / 5 5 8}$ & 8 & 96 & $\mathbf{1 8 7 4 / 1 3 3 4}$ & 15 & $\mathbf{0 . 9 9 8 3 0 2 1 6 7 / 0 . 9 9 6 6 0 3 8 9 4 0 3 1 2 8}$ \\
\hline \hline $70 / 200$ & $+0.03 / 0$ & 2.47 & $\mathbf{6 3 / 3 2}$ & $\mathbf{1 1 1 6 / 5 5 8}$ & 7 & 84 & $\mathbf{1 8 8 0 / 1 3 2 2}$ & 15 & $\mathbf{0 . 9 9 8 4 7 6 0 6 3 / 0 . 9 9 6 9 5 1 8 7 1 6 5 7 7 5 4}$ \\
\hline \hline $75 / 200$ & $+0.03 / 0$ & 2.5 & $\mathbf{6 3 / 3 2}$ & $\mathbf{1 1 1 6 / 5 5 8}$ & 7 & 84 & $\mathbf{1 8 8 0 / 1 3 2 2}$ & 16 & $\mathbf{0 . 9 9 8 6 2 3 6 / 0 . 9 9 7 2 4 7 0 8 9 2 6 2 6 1 3}$ \\
\hline \hline $80 / 200$ & $+0.03 / 0$ & 2.52 & $\mathbf{6 4 / 3 2}$ & $\mathbf{1 1 3 4 / 5 5 8}$ & 6 & 72 & $\mathbf{1 8 8 6 / 1 3 1 0}$ & 17 & $\mathbf{0 . 9 9 8 7 5 / 0 . 9 9 7 5}$ \\
\hline \hline $85 / 200$ & $+0.035 / 0$ & 2.55 & $\mathbf{6 5 / 3 3}$ & $\mathbf{1 1 5 2 / 5 7 6}$ & 6 & 72 & $\mathbf{1 9 0 4 / 1 3 2 8}$ & 17 & $\mathbf{0 . 9 9 8 8 5 9 2 2 3 / 0 . 9 9 7 7 1 8 5 3 3 3 4 2 8 6 3}$ \\
\hline \hline $90 / 200$ & $+0.035 / 0$ & 2.57 & $\mathbf{6 5 / 3 3}$ & $\mathbf{1 1 5 2 / 5 7 6}$ & 5 & 60 & $\mathbf{1 8 9 2 / 1 3 1 6}$ & 18 & $\mathbf{0 . 9 9 8 9 5 4 3 2 5 / 0 . 9 9 7 9 0 8 8 0 5 0 3 1 4 4 7}$ \\
\hline \hline $95 / 200$ & $+0.035 / 0$ & 2.6 & $\mathbf{6 6} / 33$ & $\mathbf{1 1 7 0 / 5 7 6}$ & 5 & 60 & $\mathbf{1 9 1 0 / 1 3 1 6}$ & 18 & $\mathbf{0 . 9 9 9 0 3 7 6 9 7 / 0 . 9 9 8 0 7 5 6 0 1 3 7 4 5 7}$ \\
\hline \hline $100 / 200$ & $+0.035 / 0$ & 2.62 & $\mathbf{6 7 / 3 4}$ & $\mathbf{1 1 8 8 / 5 9 4}$ & 4 & 48 & $\mathbf{1 9 1 6 / 1 3 2 2}$ & 18 & $\mathbf{0 . 9 9 9 1 1 1 2 3 5 / 0 . 9 9 8 2 2 2 7 1 7 1 4 9 2 2 1}$ \\
\hline
\end{tabular}

С увеличением диаметра протягиваемого отверстия в заготовках из легированной стали и серого чугуна на 5 мм припуск на обработку увеличивается на $0.2-0.3$ мм. Количество зубьев калибрующей части, длина калибрующей части и количество стружкоразделительных канавок цилиндрической протяжки при обработке стали и чугуна изменяются одинаково. Количество режущих зубьев и длину режущей части протяжки при обработке отверстий в чугунных заготовках рекомендуется уменьшать в два раза, по сравнению с одноименными параметрами протяжки для обработки отверстий в стальных заготовках. Для уменьшения трения на боковых сторонах режущих зубьев протяжки делают поднутрение, образуемое вспомогательным углом. Этот угол выполняется в пределах $0.993 \ldots 0.999$ град.

\section{Заключение}

На основании проведенного расчета конструктивных элементов и геометрических параметров цилиндрической протяжки и последующего анализа результатов можно сделать следующие выводы:

1. С увеличением прочности материала заготовки, подачу, допустимую по силе резания уменьшают: на $20 \%$ - для углеродистой стали, на $45 \%$ - для легированной стали (сравнение с серым чугуном).

2. При увеличении подачи, допустимой по силе резания, количество калибрующих зубьев (3) и длина калибрующей части (36 мм) цилиндрической протяжки не изменяются. С уменьшением подачи, допустимой по силе резания, количество калибрующих зубьев и длина калибрующей части изменяются с 8 до 4 и с 96 мм до 48 мм соответственно.

\section{References:}

1. (2016) Broaching (metalworking). Available: https://en.wikipedia.org/wiki/Broaching_(metal working) (Accessed: 31.10.2016).

2. (2016) Protyazhki dlya obrabotki otverstii. Available:

http://www.info.instrumentmr.ru/protiagki2.sht ml (Accessed: 31.10.2016).

3. Kupriyanova OP, Kopeykin EA (2012) Optimization of design parameters of round broaching. Bulletin of Peoples' Friendship University of Russia. Series: Engineering researches, № 1. - pp. $41-45$.

4. (1990) GOST 28442-90. Broaches for cylindrical, slitting and polyhedral profile holes. Specifications.

5. (1970) GOST 4044-70. Round shanks for broaches. Typs and main dimensions.
6. Katsev PG (1968) Broaching works. A study guide for individual and team training of workers on production. Publishing a second, revised and enlarged. M.: «High school». -247 p.

7. (1989) GOST 25346-89. Basic norms of interchangeability. Unified system of tolerances and fits. General, series of tolerances and fundamental deviations.

8. (2016) Shemy rezaniya i forma rezhushchih kromok protyazhek. Available: http://instrrez.ru/osnovy-proektirovaniyarezhushchego-instrumenta/5-shemy-rezanija-iforma-rezhushhih-kromok.html (Accessed: 31.10.2016). 